\title{
Liquid pair correlations in four spatial dimensions: Theory versus simulation
}

\author{
M. Heinen, ${ }^{1}$ J. Horbach, ${ }^{2}$ and H. Löwen ${ }^{2}$ \\ ${ }^{1}$ Division of Chemistry and Chemical Engineering, \\ California Institute of Technology, Pasadena, California 91125, USA. \\ ${ }^{2}$ Institut für Theoretische Physik II, Weiche Materie, \\ Heinrich-Heine-Universität Düsseldorf, 40225 Düsseldorf, Germany
}

\begin{abstract}
Using liquid integral equation theory, we calculate the pair correlations of particles that interact via a smooth repulsive pair potential in $d=4$ spatial dimensions. We discuss the performance of different closures for the Ornstein-Zernike equation, by comparing the results to computer simulation data. Our results are of relevance to understand crystal and glass formation in high-dimensional systems.
\end{abstract}

\section{INTRODUCTION}

Particle-resolved structure in a classical homogeneous bulk liquid is typically measured in terms of pair correlation functions. In real space, the pair correlations provide the conditional probability density to find a particle at a distance $r$ from another particle. The associated Fourier transform correlates density waves of wavenumber $k$ [1]. While the latter is a typical outcome of a scattering experiment [2], the former can be obtained from the real-space coordinates of the individual particles such as colloids [3, 4] or dusty plasmas [5]. Computer simulations of classical many-body systems with a prescribed particle pair-interaction potential are a suitable and well-established route to calculate pair correlations [6]. There are, however, situations that require a (semi-)analytical statistical mechanical approach as an alternative to computer simulations. In such cases, there is a choice of various liquid integral equations that are based on the Ornstein-Zernike equation, and that have often been proven to predict pair correlations accurately and efficiently [1, 2, 7 13]. The large body of complementary experiments, simulations and analytical theory have resulted in a good understanding of pair correlations in the fluid or liquid state by now.

Most of the studies so far have focused on the physically most relevant situation of three spatial dimensions $(d=3)$, but liquids can also exist in lower spatial dimensions when they are confined [14, 15], e.g., to twodimensional interfaces [16] or between plates 17] $(d=2)$ or inside narrow cylindrical tubes $(d=1)$ [18]. Though they do not possess an immediate physical realization, higher spatial dimensions $(d>3)$ have been another focus of recent research. The motivation to consider dimensions higher than three derives from the ambition to understand the salient necessary ingredients for freezing and the glass transition. In the existing literature on higher dimensional particulate systems, hard hyperspheres have mostly been studied [19 23] while there are less studies for particles with soft pair potentials like, e.g., the Lennard-Jones potential [24, 25].

The computational effort for particle-resolved computer simulations rises quickly as a function of $d$, which effectively limits computer simulations to dimensions $d \lesssim 12$ [26]. Analytical [27-29] or efficient numerical [11] methods for the calculation of pair correlations in dimensions $d>3$ are therefore worth aspiring for. In the present study, we examine the accuracy of the hypernetted chain (HNC) 30], Percus-Yevick (PY) 31], and Rogers-Young (RY) 32 integral equations, which are compared with numerically accurate computer simulations of particles with soft interactions of the WeeksChandler-Andersen (WCA) 33] type, in $d=4$ spatial dimensions. We find that (just like in $d=3$ spatial dimensions) the RY scheme predicts pair-correlations in excellent agreement with the computer simulation results, while the PY and HNC scheme show severe over- and under-estimation of the undulations in the static structure factor, respectively.

The remaining parts of this article are organized as follows: In section \we define the WCA fluid under study. We continue in section III to outline the computer simulations, and in section IV the liquid integral equations that we use to compute particle pair correlations. Results for the static structure factor are presented in section $\mathrm{V}$. which is followed by our concluding remarks given in section VI

\section{WEEKS-CHANDLER-ANDERSEN PAIR POTENTIAL}

We study homogeneous fluids of spherically symmetric, monodisperse particles that interact via a smoothed Weeks-Chandler-Andersen (WCA) potential [33], i.e., a Lennard-Jones potential of depth $\varepsilon$, which has been truncated at the minimum position $r=r_{c}=2^{1 / 6} \sigma$, and shifted upwards by $\varepsilon$. Thus, this non-negative (repulsive) pair potential is defined by

$$
u(r)= \begin{cases}0 & \text { for } r>r_{c}=2^{1 / 6} \sigma, \\ f(r)\left[4 \varepsilon\left(\left(\frac{\sigma}{r}\right)^{12}-\left(\frac{\sigma}{r}\right)^{6}\right)+\varepsilon\right] \quad \text { otherwise, }\end{cases}
$$


where $f(r)=\left(r-r_{c}\right)^{4} /\left[(\sigma / 200)^{4}+\left(r-r_{c}\right)^{4}\right]$ is a smoothing function that decays rapidly from $f(r) \approx 1$ for $r<r_{c}-\sigma / 200$ to $f(r)=0$ for $r=r_{c}$. The function $f(r)$ provides continuity of forces at $r_{c}$ and thus, in a molecular dynamics simulation, a better numerical stability is achieved when using this smoothing function.

The thermodynamic equilibrium state of the WCA fluid studied here is fully described by two dimensionless parameters: The normalized thermal energy $k_{B} T / \epsilon$ (with Boltzmann constant $k_{B}$ ) and $n \sigma^{d}$, which is the number of particles in a $d$-dimensional volume $\sigma^{d}$. Here, $n=N / L^{d}$ is the number density for $N$ particles in a hypercubic box of edge length $L$ (taken in the thermodynamic limit $N \rightarrow \infty$ and $L \rightarrow \infty$, where $n$ is held fixed). Note that in the limiting case of vanishing temperature $(T \rightarrow 0$ or $\epsilon \rightarrow \infty)$, the smoothed WCA potential in Eq. (11) reduces to the pair-potential of hard spheres with diameter $r_{c}$.

\section{COMPUTER SIMULATIONS}

We performed molecular dynamics (MD) simulations of a four-dimensional, monodisperse system of 20000 particles that interact via the WCA potential, as given by Eq. (11). Newton's equations of motion were integrated with the velocity form of the Verlet algorithm using a time step of $\delta t=0.00072$ in units of $\tau=\sqrt{m \sigma^{2} / \varepsilon}$ (with mass $m=1.0$ ). The particles were put into a simulation box with linear dimension $L=10.511205 \sigma$, applying periodic boundary conditions in all four spatial directions. Simulations were done at the temperatures $T=1.66,1.7,1.8,1.85,1.9,2.0,2.5,4.0,7.0$ (in units of $\varepsilon\left(k_{B}\right)$. At each temperature, the system was fully equilibrated, requiring equilibration runs between $10^{5}$ time steps at $T=7.0 \varepsilon / k_{B}$ and $4 \times 10^{7}$ time steps at $T=1.66 \varepsilon / k_{B}$. The equilibration runs were followed by production runs of double length, from which the structure factor $S(q)$ was computed. During equilibration, temperature was fixed by periodically coupling the system to a stochastic heat bath. The production runs were done in the microcanonical ensemble. Note that none of the runs showed any sign of crystallization.

\section{LIQUID INTEGRAL EQUATIONS}

The Ornstein-Zernike equation for homogeneous and isotropic, $d$-dimensional fluids reads

$$
h(r)=c(r)+n \int d^{d} r^{\prime} c\left(r^{\prime}\right) h\left(r-r^{\prime}\right)
$$

in terms of the $d$-dimensional particle number density and the total and direct correlation functions $h(r)$ and $c(r)$, respectively [1]. To obtain a closed integral equation for a given kind of pair-potential $u(r)$, the OrnsteinZernike equation must be supplemented by a closure relation. With the exception of very small number densities, exact closure relations are unknown in general and one has to resort to approximate closures. Here we study three different approximate closures. The first two are the PY closure 31]

$$
c(r)=[\gamma(r)+1] \times\left[e^{-\beta u(r)}-1\right]
$$

and the HNC closure 30]

$$
c(r)=-\gamma(r)-1+e^{\gamma(r)-\beta u(r),}
$$

both written in terms of the indirect correlation function $\gamma(r)=h(r)-c(r)$ and the inverse thermal energy $\beta=1 /\left(k_{B} T\right)$. Both the PY closure and the HNC closure are thermodynamically inconsistent, in the sense that the predicted normalized inverse isothermal osmotic compressibility computed in the fluctuation route,

$$
\frac{1}{\chi_{c}}=\beta\left(\frac{\partial P_{c}}{\partial n}\right)_{T}=1-n \int_{0}^{\infty} c(r) d r,
$$

does not match the corresponding expression

$$
\frac{1}{\chi_{v}}=\beta\left(\frac{\partial P_{v}}{\partial n}\right)_{T}
$$

from the virial route. Here, $P_{v}$ is the virial pressure which, for monodisperse particles with WCA pairpotentials as studied here, can be calculated according to

$$
\frac{\beta P_{v}}{n}= \begin{cases}1+\frac{\beta n \omega_{d}}{2 d} r_{c}^{d} g\left(r_{c}^{+}\right) & \text {for } T=0, \\ 1-\frac{\beta n \omega_{d}}{2 d} \int_{0}^{\infty} d r r^{d} g(r) \frac{d u(r)}{d r} . & \text { for } T>0 .\end{cases}
$$

In Eq. (7), $g(r)=h(r)+1$ is the radial distribution function, $\omega_{d}=2 \pi^{d / 2} / \Gamma(d / 2)$ is the $d$-dimensional unit hypersphere surface in terms of the Gamma function $\left(\omega_{4}=2 \pi^{2}\right)$, and $g\left(r_{c}^{+}\right)=\lim _{r}{ }_{r} g(r)$ is the contact value of the hard-sphere radial distribution function in the special case of $T=0$.

Thermodynamic inconsistency with respect to the isothermal compressibility can be avoided by using the RY closure [32]

$$
c(r)=-\gamma(r)-1+e^{-\beta u(r)}\left[1+\frac{e^{\gamma(r) f(r)}-1}{f(r)}\right],
$$

where $f(r)=1-\exp \{\alpha r\}$ is a mixing function that depends on the non-negative inverse length $\alpha$. The RY closure interpolates between the PY closure (which is recovered in both limits $r \rightarrow 0$ and $\alpha \rightarrow 0$ ), and the HNC closure (recovered for $r \rightarrow \infty$ or $\alpha \rightarrow \infty$ ). The parameter $\alpha$ is selected such that equal values are obtained for the isothermal osmotic compressibility calcu- 


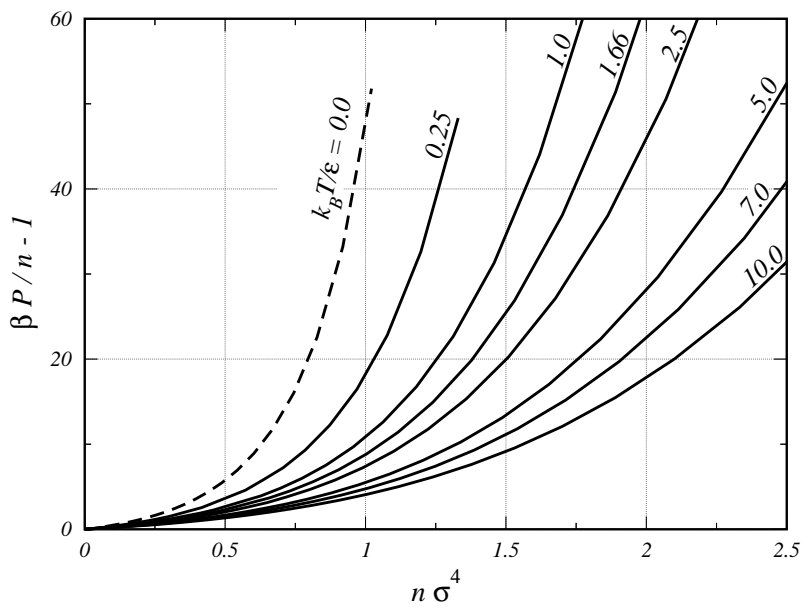

Figure 1. Equations of state for four-dimensional WCA fluids, for eight different reduced temperatures $k_{B} T / \epsilon=$ $0.0,0.25,1.0,1.66,2.5,5.0,7.0$, and 10.0 , as indicated. The Rogers-Young normalized excess pressure is plotted as a function of the number of particles in a volume $\sigma^{4}$. The dashed curve for $T=0.0$ is the result for a four-dimensional fluid of hard spheres with diameter $r_{c}=2^{1 / 6} \sigma$.

lated in the fluctuation route and the virial route. The standard RY scheme, as used in the present work, is thermodynamically self-consistent with respect to the isothermal osmotic compressibility only. At the expense of an increased numerical effort, the RY scheme can be further improved by requiring consistency in additional, independent thermodynamic quantities [12]. Note also that the RY scheme usually does not have a solution for non-positive-definite pair potentials. However, for such potentials different thermodynamically partially self-consistent closure relations have been devised [34, 35], similar in spirit to the RY scheme.

The equation of state of four-dimensional WCA fluids at various temperatures is investigated in Fig. 1, where we plot the excess part of the normalized pressure, $\beta P / n-1$, as calculated in the RY scheme.

The (partial) thermodynamic self consistency of the RY scheme usually results in a significantly improved accuracy of the pair-correlation functions including $g(r)$ and the static structure factor $S(q)=1+n \mathcal{F}[h(r)](q)$, with $\mathcal{F}$ denoting the $d$-dimensional Fourier transform of an isotropic function. However, the good accuracy of the RY scheme is essentially an empirical finding that should be tested by comparison to simulation results, for each pair-potential and each number of spatial dimensions.

In the important, generic case of hard hyperspheres, the (in this case rather accurate) PY integral equation [31] can in principle be solved analytically for arbitrary odd dimension [27, 28], and semi-analytically for arbitrary even dimension [29]. However, these (semi-)analytical solution methods are quite cumbersome, with an analytical effort that rises quickly with increasing number of dimensions $d$. Moreover, the PY scheme usually over-estimates the undulations in the pair-correlation functions (in particular in the static structure factor), when it is applied to soft repulsive pairpotentials. In case of soft particle interactions, like studied in the present work, analytical progress can be made, e.g., by resorting to the mean spherical approximation (MSA) [1] which represents a closure for the OrnsteinZernike equation that results in a linear integral equation. Remedy for the unsatisfactory accuracy of the MSA has been proposed in several studies [10, 36, 37], in form of semi-analytical rescaling arguments.

A versatile and computationally efficient alternative to the (semi-)analytical solution of arbitrary-dimensional liquid integral equations is the numerical solution by means of a spectral solver. Within this numerical method, employed in the present study, it is easy to implement a variety of different closures for the OrnsteinZernike equation, suitable for a variety of particle pairinteraction potentials. In the present work, we employ a numerical method that we have comprehensively outlined in Ref. 11. This method, based on techniques that were originally published in Refs. [38 42], is applicable in all positive spatial dimensions $d$ and is numerically very efficient and robust. Our implementation of the numerical solution algorithm allows to compute solutions for $1 \leq d \lesssim 30$, the upper boundary for $d$ depending on the kind of pair-potentials, the closure relation, and the particle number density.

As a further motivation for studying liquid integral equations in higher dimensions, we note here that modecoupling theory (MCT) has been employed to study the glass transition of hard hyperspheres in very high dimensions [43]. In Ref. [43], the structural input to the $\mathrm{MCT}$ equations was generated by approximating $c(r)$ by the Mayer function $\exp \{-\beta u(r)\}-1$. As outlined in Ref. [43], the latter approximation is exact in the limit of infinite spatial dimension $(d \rightarrow \infty)$, and remains to be a good approximation of the actual particle pair correlations for dimensions $d \gtrsim 100$. For dimensions $d$ in the range $1 \leq d \lesssim 100$, the approximation $c(r) \approx \exp \{-\beta u(r)\}-1$ is insufficient unless the particle number density is very low. This leads to unphysical artifacts in the predicted glass transition lines for $d \lesssim 100$ [43]. As an alternative, one can use simulation results for the static pair correlations as input to $\mathrm{MCT}$ 44]. However, the computational effort of computer simulations rises quickly as a function $d$, which limits this approach to dimensions $d \lesssim 12$ [26]. Hence there is a gap for $10 \lesssim d \lesssim 100$, where neither simulation results nor infinite-dimensional limiting expressions can be used. This gap can be essentially filled in by numerical liquid integral equation solutions as reported in the present article and in Ref. [11]. 


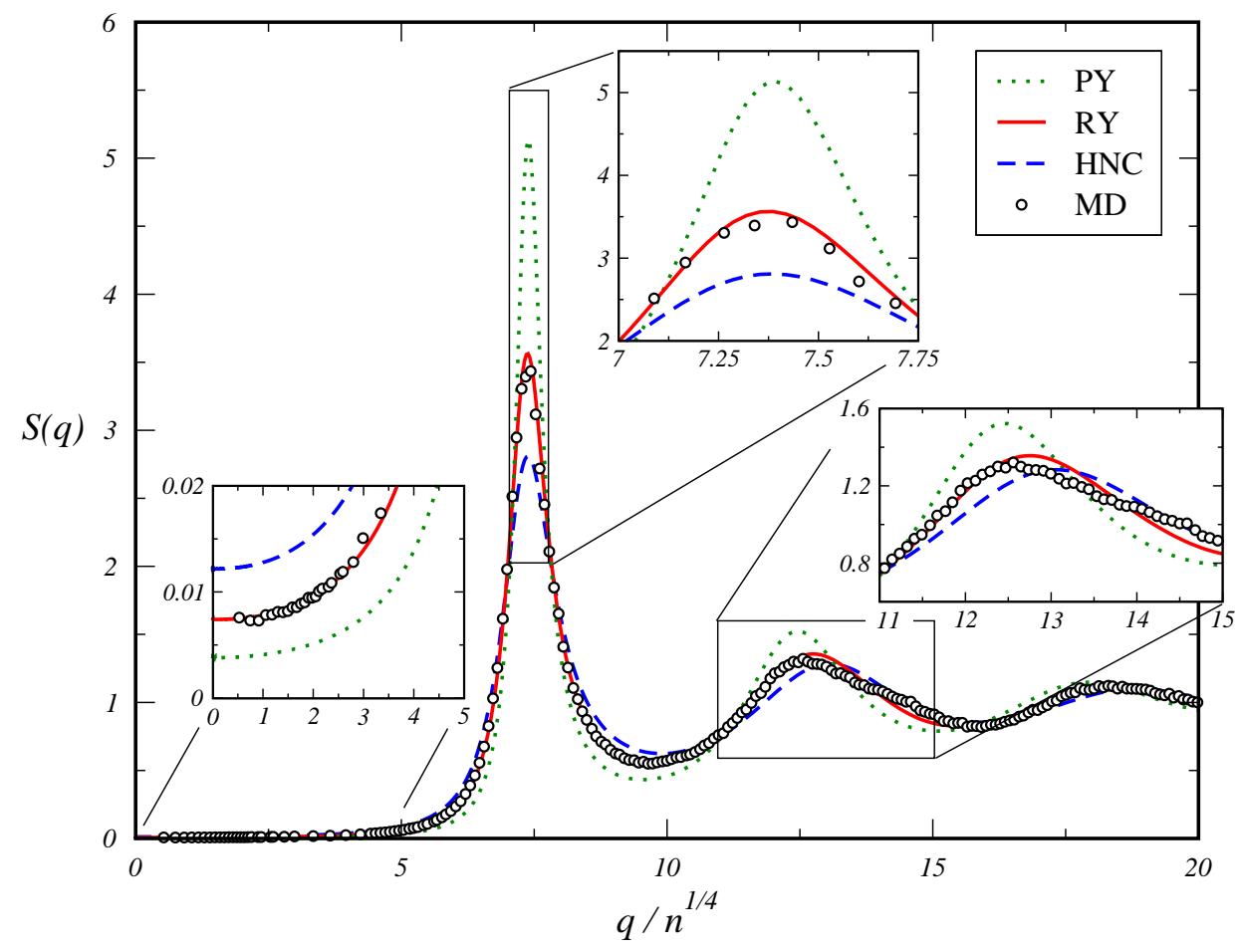

Figure 2. Static structure factor for a four dimensional fluid of particles interacting via WCA pair potentials [Eq. (10)], for particle number density $n=1.6384 \sigma^{-4}$ and for $k_{B} T=1.66 \varepsilon$. Black circles filled in white: Molecular dynamics simulation results. Dotted green curve: Percus-Yevick scheme. Solid red curve: Rogers-Young scheme. Blue dashed curve: Hypernetted chain scheme. The three insets magnify the region of very low wave numbers $q$, the region around the principal peak, and around the second peak.

\section{STATIC STRUCTURE FACTORS}

In Fig. 2 we plot the static structure factor, $S(q)$, for a four-dimensional WCA fluid at a rather high number density $n=1.6384 \sigma^{-4}$, and a rather low temperature, $T=1.66 \varepsilon / k_{B}$. Under these conditions the fluid exhibits very pronounced pair correlations. Shown are the results from our Molecular Dynamics simulation (black circles filled in white), and from the HNC (blue dashed curve), RY (red solid curve), and PY (green dotted curve) integral equations. Three insets magnify the regions of very low wave numbers, $q \gtrsim 0$, the region around the structure factor's principal peak, and the region around the second peak. Note that the HNC scheme predicts a structure factor with considerably underestimated undulations, and that the PY scheme is severely over-estimating these undulations, while the RY scheme is in very good (if not excellent) agreement with the simulation result. Each of these observations is in-line with the usual observations that have been made for three-dimensional fluids of purely repulsive particles.

The only obvious difference between the MD structure factor and the RY structure factor (and the PY and HNC results alike) is a failure of the liquid integral equation schemes to predict the shape of the second peak in $S(q)$ :
The right flank of the second peak in the simulation result exhibits a nearly linear decay of $S(q)$ for values of $q / n^{1 / 4}$ between 12.5 and 15 . This feature is missing in each of the liquid integral equation scheme results, which predict a rounder shape of the second peak. Similar features in the second peak of the structure factor have been discussed as possible freezing precursors, and as signatures of short-ranged order in the liquid phase [45, 46] (see also the related Ref. [47]). To the best of our knowledge, the second peak shape feature is not observed in any of the usual liquid integral equation schemes that are formulated on the level of pair-correlation functions. A similar feature (in the radial distribution function, however, and for $d=3$ ) has been reported in Ref. [13], where a computationally more sophisticated integral equation scheme was solved that includes non-trivial triplet correlations. The implementation of such a scheme for the four-dimensional fluids under investigation is beyond the scope of the present work.

Note from Fig. 3, that the agreement between the MD simulation and RY-scheme structure factors is very good for higher temperatures $\left(T=2.5 \varepsilon / k_{B}\right.$ in the left panel of Fig. 3, and $T=7.0 \varepsilon / k_{B}$ in the right panel). For $T=7.0 \varepsilon / k_{B}$, the flattened second peak feature has practically disappeared in the MD simulation results, and the agreement to the RY scheme is almost perfect. 

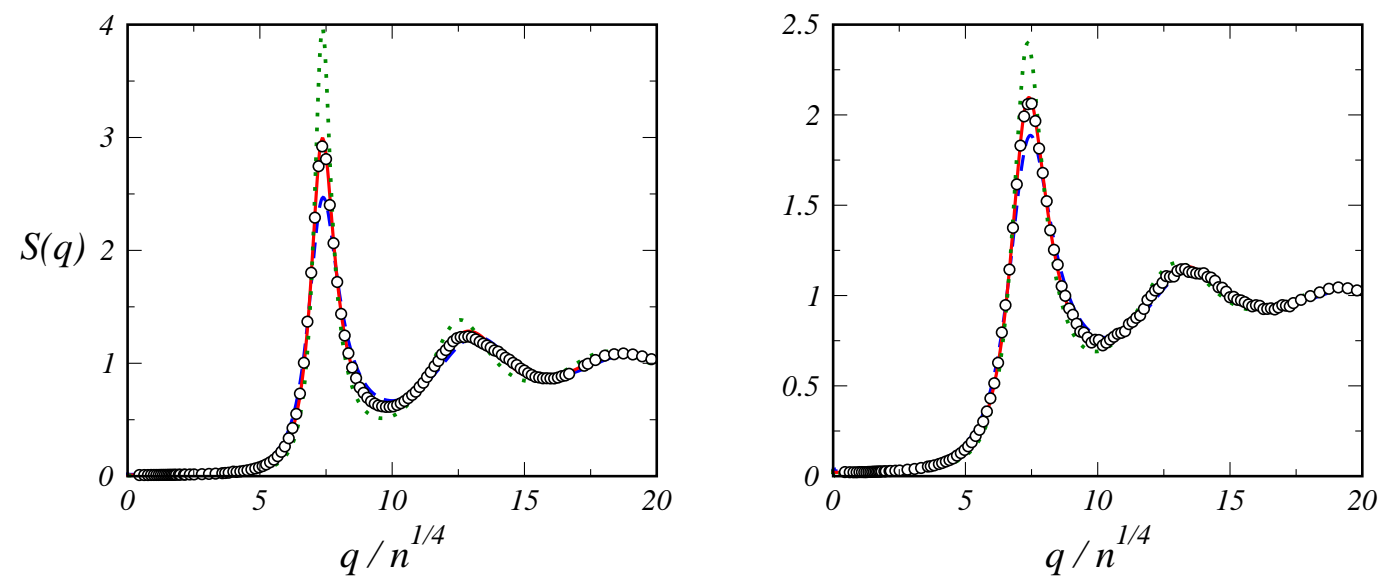

Figure 3. Static structure factors for two four-dimensional fluids of particles interacting via WCA pair potentials [Eq. [10], for dimension $d=4$, particle number density $n=1.6384 \sigma^{-4}$, and for $k_{B} T=2.5 \varepsilon$ (left panel) and $k_{B} T=7.0 \varepsilon$ (right panel). Black circles filled in white: Molecular dynamics simulation results. Dotted green curves: Percus-Yevick scheme. Solid red curves: Rogers-Young scheme. Blue dashed curves: Hypernetted chain scheme.

\section{CONCLUSIONS}

We have demonstrated that the RY integral equation scheme predicts pair correlations in homogeneous fourdimensional fluids of particles with soft repulsive interactions in very good agreement with numerically accurate, but computationally expensive Molecular Dynamics simulations. This finding, which is in line with the known excellent performance of the RY scheme for threedimensional fluids, qualifies the RY scheme as a numerically highly efficient method for calculating the structure input that is needed for theories of dynamics, phase behaviour and vitrification in higher dimensions [43]. Despite its overall very good accuracy, the RY scheme (as well as the PY and HNC schemes) fails to predict the correct shape of the second peak in the static structure factor when the particle repulsion becomes very strong. We expect that inclusion of nontrivial triplet correlations into a (thermodynamically partially self-consistent) liq- uid integral equation scheme [13] for arbitrary spatial dimensions could result in an improved ability of the theory to reproduce the static structure factor, particularly around its second peak.

\section{ACKNOWLEDGEMENTS}

It is our pleasure to thank Ramón Castañeda-Priego, Mauricio D. Carbajal-Tinoco and Cristian Vasile Achim for helpful comments and discussions. M.H. acknowledges support by a fellowship within the PostdocProgram of the German Academic Exchange Service (DAAD). J.H. acknowledges funding via the DFG Research Unit FOR 1394 "Nonlinear Response to Probe Vitrification". M.H. and H.L. acknowledge funding within the ERC Advanced Grant INTERCOCOS (project number 267499).
[1] J.P. Hansen and I.R. McDonald, Theory of Simple Liquids, 3rd ed. (Academic Press, London, 1986).

[2] F. Westermeier, B. Fischer, W. Roseker, G. Grübel, G. Nägele and M. Heinen, J. Chem. Phys. 137, 114504 (2012).

[3] C.P. Royall, M.E. Leunissen, A.P. Hynninen, M. Dijkstra and A. van Blaaderen, J. Chem. Phys. 124, 244706 (2006).

[4] L. Assoud, F. Ebert, P. Keim, R. Messina, G. Maret and H. Löwen, J. Phys.-Condes. Matter 21, 464114 (2009).

[5] A. Ivlev, H. Löwen, G. Morfill and C.P. Royall, Complex Plasmas and Colloidal Dispersions: Particle-resolved Studies of Classical Liquids and Solids, Series in Soft Condensed Matter, Vol. 5 (World Scientific, Singapore, 2012).
[6] M.P. Allen and D.J. Tildesley, Computer Simulation of Liquids (Oxford University Press, Oxford OX2 6DP, 1991).

[7] G. Nägele, Phys. Rep. 272, 216 (1996).

[8] C. Caccamo, Phys. Rep. 274, 1 (1996).

[9] A. Parola and L. Reatto, Adv. Phys. 44, 211 (1995).

[10] M. Heinen, P. Holmqvist, A.J. Banchio and G. Nägele, J. Chem. Phys. 134, 044532, ibid. 129901 (2011).

[11] M. Heinen, E. Allahyarov and H. Löwen, J. Comput. Chem. 35, 275 (2014).

[12] M.D. Carbajal-Tinoco, J. Chem. Phys. 128, 184507 (2008).

[13] J.M. Brader, J. Chem. Phys. 128, 104503 (2008).

[14] C. Alba-Simionesco, B. Coasne, G. Dosseh, G. Dudziak, K.E. Gubbins, R. Radhakrishnan and M. Sliwinska- 
Bartkowiak, J. Phys.-Condes. Matter 18, R15 (2006).

[15] H. Löwen, J. Phys.-Condes. Matter 21, 474203 (2009).

[16] K. Zahn, R. Lenke and G. Maret, Phys. Rev. Lett. 82, 2721 (1999).

[17] M. Schmidt and H. Löwen 55, 7228 (1997).

[18] E.C. Oğuz, R. Messina and H. Löwen, EPL 94, 28005 (2011).

[19] M. Baus and J.L. Colot, Phys. Rev. A 36, 3912 (1987).

[20] M. González-Melchor, J. Alejandre and M. López de Haro, J. Chem. Phys. 114, 4905 (2001).

[21] M. Skoge, A. Donev, F.H. Stillinger and S. Torquato, Phys. Rev. E 74, 041127 (2006).

[22] R. Finken, M. Schmidt and H. Löwen, Phys. Rev. E 65, 016108 (2002).

[23] J.A. van Meel, B. Charbonneau, A. Fortini and P. Charbonneau, Phys. Rev. E 80, 061110 (2009).

[24] J.D. Eaves and D.R. Reichman, PNAS 106, 15171 (2009).

[25] R. Brüning, D.A. St-Onge, S. Patterson and W. Kob, J. Phys.-Condes. Matter 21, 035117 (2009).

[26] P. Charbonneau, A. Ikeda, G. Parisi and F. Zamponi, Phys. Rev. Lett. 107, 185702 (2011).

[27] M. Robles, M. López de Haro and A. Santos, J. Chem. Phys. 120, 9113 (2004).

[28] R.D. Rohrmann and A. Santos, Phys. Rev. E 76, 051202 (2007).

[29] M. Adda-Bedia, E. Katzav and D. Vella, J. Chem. Phys. 129, 144506 (2008).

[30] T. Morita, Prog. Theo. Phys. 20, 920 (1958).
[31] J.K. Percus and G.J. Yevick, Phys. Rev. 110, 1 (1958).

[32] F.J. Rogers and D.A. Young, Phys. Rev. A 30, 999 (1984).

[33] J.D. Weeks, D. Chandler and H.C. Andersen, J. Chem. Phys. 54, 5237+ (1971).

[34] G. Zerah and J.P. Hansen, J. Chem. Phys. 84, 2336 (1986).

[35] J.M. Bomont and J.L. Bretonnet, J. Chem. Phys. 121, 1548 (2004).

[36] J.P. Hansen and J.B. Hayter, Mol. Phys. 46, 651 (1982).

[37] I.K. Snook and J.B. Hayter 8, 2880 (1992).

[38] K.C. Ng, J. Chem. Phys. 61, 2680 (1974).

[39] J.D. Talman, J. Comput. Phys. 29, 35 (1978).

[40] P.J. Rossky and H.L. Friedman, J. Chem. Phys. 72, 5694 (1980).

[41] A.J.S. Hamilton, Mon. Not. R. Astron. Soc. 312, 257 (2000).

[42] A. J. S. Hamilton's FFTLog website http://casa.colorado.edu/ ajsh/FFTLog/

[43] B. Schmid and R. Schilling, Phys. Rev. E 81, 041502 (2010).

[44] P. Charbonneau, A. Ikeda, J.A. van Meel and K. Miyazaki, Phys. Rev. E 81, 040501 (2010).

[45] T. Schenk, D. Holland-Moritz, V. Simonet, R. Bellissent and D.M. Herlach 80 (2002).

[46] D. Holland-Moritz, O. Heinen, R. Bellissent and T. Schenk, Mater. Sci. Eng. A 449, 42 (2007).

[47] J. Gapinski, G. Nägele and A. Patkowski, J. Chem. Phys. 141, 124505 (2014). 